Aus der Eermatologischen Abteilung des Städtischen Rudolf Virchow-Krankenhauses in Berlin.

(Dirigierender Arzt: Prof. A. Buschke.)

\title{
Komplikationen und Heilungen subakuter Leberatrophie bei Syphilis.
}

Von Prof. Dr. A. Buschke und Dr. E. Langer, Assistenzarzt.

In den letzten Jahren, besonders durch das gehäufte Auftreten der Leberatrophic am Ende des Krieges und in der Nachkriegszeit, ist so oft über die typisch verlaufenen Fälle gesprochen und geschrieben worden, daß es zur Zeit wohl nicht lohnend wäre, einen weiteren Beitrag hierzu zu liefern, zumal, soweit unsere Forschungen auf diesem Gebiete gedrungen sind, das typische Bild der Leberatrophie sowohl in klinischer wie in pathologischer Hinsicht abgerundet ist. Allein der ätiologische Faktor wird noch vielfach diskutiert, doch dürfte es auch hierin zur Zeit unmöglich sein, aus den vielen ätiologischen Momenten, die in der Hauptsache auf hypothetischen Annahmen beruhen, eine einheitliche Ursache für dieses Leiden herauszuschälen. Mañ muß doch woh! unter den gegebenen. Verhältnissen annehmen, dab es vielerlei schädliche Momente sind, die auf den verschiedensten Wegen zu derselben Erkrankung führen, soda $\beta$ wohl jeder für seinen Teil mit seiner Theorie Recht behält. $\mathrm{Ob}$ wir eine hämatogene oder eine enterogene Noxe annehmen, ob wir die Ursache für infektiös oder toxisch halten, für jeden Fall werden sich beweiskräftige Momente heranziehen lassen. Dazu kommt, daB unsere Leberuntersuchungsbzw. Funktionsprüfungen doch recht grob und unzulänglich sind und wir im allgemeinen zur Deutung des Krankheitsbildes und der Ursachen desselben auf theoretische Annahmen und Vergleiche mit ähnlichen Fällen angewiesen sind.

Gegenüber dem recht einförmigen Bilde der typischen Fälle von akuter Leberatrophie sind es die abortiv verlaufenden und die Kompli- 
kationen, und bei letzteren besonders die in klinische Ausheilung übergehenden Fälle, die uns in der Pathogenese fördern, uns durch die Mannigfaltigkeit ihres Krankheitsbildes interessieren und uns ganz neue Fragen für den Ablauf der Lebererkrankungen vorlegen. Seit von $\mathrm{Huber}$ und $\mathrm{Ka}$ a s $\mathrm{ch}$, Strau $B$, Umber u. a. nach dem Kriege die ersten Falle von intra vitam diagnostizierter und $z$. $T$. von klinisch ausgeheilter, mit Aszites einhergehender Leberatrophie veröffentlicht sind, hat die Beschreibung dieses Krankheitsbildes mannigfache Wiederholung erfahren, wobei es sich herausstellte, daB die mit Aszites einhergehenden Fälle gar nicht so selten sind, wie es $z$. B. $\mathrm{U} \mathrm{mb}$ e $\mathrm{r}$ anfänglich angenommen hatte, soda $\mathrm{H}$ anser $u$. a. wohl schon mit einem gewissen Recht behaupten können, daß der Aszites in das Schulbild der Leberatrophie hineingehöre. Und zwar teilen fas alle Autoren die Ansicht, daB es die protrahiert und oftmals die mit Rezidiven verlaufenden Fälle sind, in denen sich der Aszites einstellt (Hart, Seyfarth, Strümpell). Die Ursache des Aszites wird auf die Leberveränderungen zurückgeführt, durch die es infolge der interstitiellen Bindegewebswucherungen innerhalb und an der Peripherie der Azini zu Stauung im Pfortaderkreislauf durch Zugrundegehen der Pfortaderkapillaren kommt. Aber auch schon ältere Autoren, wie z. B. Thierfelder, haben auf das Vorhandensein des Aszites bei manchen Leberatrophiefällen aufmerksam gemacht und außerdem die Beobachtung mitgeteilt, die in keiner Arbeit der letzten Zeit wiederholt bzw. bestätigt ist, daB es in manchen Fällen auch $z u$ hydropischen Ansammlungen in den übrigen serösen Säcken und auch unter der Haut kommen kann. Nur letzteres wird allerdings von St ra uB berichtet. Bange erwähnt in seiner Dissertation unter der älteren, bis 1914 vorhandenen Literatur über Leberatrophie und Aszites auch diejenigen Fälle, bei denen nében dem Aszites in anderen Höhlen Ergüsse aufgetreten sind. Es sind dies die von W a ld e y e r, H lava, $M$ eder und $M$ archand auf dem Sektionstisch beobachteten Fälle. H lava zog aus seiner Beobachtung den Schluß, daß man berechtigt sei, den allgemeinen Hydrops als ein neues klinisches Symptom bei langdauernden Fällen von gelber Leberatrophie zu betrachten.

Wir selbst haben gleichzeitig zwei Fälle von gelber Leberatrophiebeis yphilis und nach Salvarsan beobachtet, die neben einem ausgedehnten Aszites Hydroth or ax a.ufwi ese $n$, der in dem einen zur Sektion gekommenen Fall autoptisch, in dem andern klinisch einwandfrei nachgewiesen werden konnte. Aus den Krankengeschichten sei in Kürze das Wichtigste hervorgehoben

1. F all. A. D., 24 jähriger Mann. Aufnahme 11. IX. 1921. Früher nie krank gewesen. April 1921 Primäraffekt. $6 \mathrm{Hg}$ und 4 Neosalvarsan. Letztes Neosalvarsan anfangs Mai 1921. Seit Ende Juni Magenbeschwerden und Gelbsucht. Bis Ende Juli diätetisch behandelt und gebessert entlassen. Seit 3 Wochen Gelbfärbung erneut autgetreten und zugenommen. Befinden verschlechtert. Seit 4 Tagen autgetriebener Leib und geschwollene Beine.

Befund: Kräftig gebauter Mann, Haut und Schleimhäute kanariengelb verfärbt, Beine stark geschwollen. Leib aufgetrieben, in den abhänngigen Partien Dämpfung bis in die Nähe des Nabels. Hier Tympanie. Leber nicht palpabel, perkutorisch nach oben verdrängt und verkleinert. Milz nicht palpabel. Lunge 1. h. u. Dämptung und abgeschwächtes Atemgeräusch. Herz: Töne leise, sonst o. B. Genitalorgane: schwere Gonorrhoe mit Prostatitis und Spermatozystitis. Urin: bierbraun verfärbt, Eiweiß, Zucker -, Sediment o. B., Bilirubin + , Urobilin - , Urobilinogen -, Leuzin und Tyrosin -.. Stuhl entfärbt, Wa.R. ++++

13. IX. täglich steigende Temperatur, zur Zeit $39^{\circ}$, große morgendliche Remission. Zunehmende Atemnot, Pleurapunktion links: Entleerung von $1200 \mathrm{ccm}$ seröser Flüssigkeit, spezifisches Gewicht 1010 , Rivalta

Diagnose: subakute Leberatrophie mit Aszites, $\mathrm{Hy}$ drothorax 1. und Anasarka.

14. IX. Temperatur bis auf 39,8 gestiegen, im Urin Eiweiß + . Sediment: granulierte Zylinder, rote und weiBe Blutkörperchen, Epithelien. Patient verfällt sehr, Hautfärbung schmutzig graugelblich, Aszitespunktion: $1200 \mathrm{ccm}$ ikterisch gefärbte klare Flüssigkeit. Patient ist leicht somnolent, liegt ruhig da, keine Krämpfe. Blutbild normal, bis auf geringe Vermehrung der Leukozyten.

17. IX. Temperatur abgefallen, Befinden sehir verschlechtert. Hydrothorax wieder vergrößert. Punktion: etwa $1200 \mathrm{ccm}$ klares Transsudat. Aszites und Anasarka bestehen weiter.

19. IX. Zunehmende Verschlechterung. Völlige Benommenheit, Exitus. Therapeutisch wurde neben Exzitantien deimal je $2 \mathrm{ccm}$ Novasurol gegeben.

Sektion: Prosektor Dr. Christeller). Beine geschwollen, ödematös. L. Pleurahöhle $700 \mathrm{ccm}$ Flüssigkeit. R. Pleurahöhle frei. Lungen und Herz o. B. Bauchhöhle: $700 \mathrm{ccm}$ Aszites. Magen und Darm o. B. Milz: $200 \mathrm{~g}$, weich. Leber $950 \mathrm{~g}$. R. Leberlappen hart, gewölbt, durch zahlreiche flache Stränge in unregelmäBige Knoten geteilt. L. Lappen auf der. Unterseite von gleichem Aussehen, oben

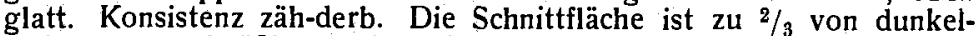
gelber, un regelmäßiger, wie Azini aussehender Zeichnung eingenommen, das übrige Drittel zeigt rötliches Gewebe mit unregelmäßiger grauer Felderung; der I. Lappen zeigt ebenfalls das Bild eines rötlichen, von grauen, unregelmäßigen Streifen durchzogenen Gewebes, Pfortader ist leer. Ductus choledochus gleichkalibrig, periportale Lymphdrüsen bilden ein dickes Paket. Nieren: o. B. Genitalorgane: Ausgedehinte Abszedierungen in den Nebenhoden, Prostata und Samenblasen.
Sektionsdiagnose: Subakute Leberatrophie, Aszites, Anasarka, Hydrothorax I., Spermatozystit is, Epididymitis, Prostatitis gonorrhoica.

2. Fall. 26 jähriger Mann. Aufnahme 18. X. 1921. Mai 1921 Gonorrhoe und Schanker. Bis Juni 12 Neosalvarsan. Wa.R. bei der Entlassung -

9. X. 1921 bemerkte Patient einen Ausschlag am Körper, gleichzeitig traten Magenbeschwerden und Erbrechen auf, Verstopfung. Erhielt am 14.X. von einem Arzt Neosalvarsan. Daraufhin leichte Gelbfärbung.

B e fund: Bei der Aufnahme am 18. X. zeigen Haut- und Schleimhäute deutlich ikterische Verfärbung, daneben makulöses Exanthem. Genitale: Gonorrhoea anterior. Bauchhöhle: Leib nicht aufgetrieben, Leber 2 Querfinger unterhalb des Rippenbogens fühlbar, druckschmerzhaft, Milz hicht verg rößert. Urin : bierbraun. Bilirubin, Urobilin +, Urobilinogen -, Leuzin und Tyrosin - Stuhl wenig gefärbt. Diagnose: Roseola. Ikterus.

27. X. In den letzten Tagen bei zunächst diätetischer Therapie Befinden verschlechtert. Leib etwas aufgetrieben. Aszites nachweisbar. Urin: Gallenfarbstoffe + , Leuzin, Tyrosin -... Stuhl fast entfärbt. Beginn einer Schmierkur $4 \mathrm{~g}$ pro die.

29. X. Befinden verschlechtert, Aszites stärker geworden. Leber nicht palpabel. Patient ist stark dyspnoisch, $r$. Lunge $h$. u. bis herau zur Mitte der Skapula völlige Dämpfung, aufgehobenes Atemgeräusch. L. Lunge nur geringe Dämpfung h. u.

B e f und röntgenologisch bestätigt. Diagnose: Aszites, Hydrothorax $r$. u. 1 .

1. XI. Hydrothorax und Aszites stärker geworden. Wegen der Verdrängungserscheinungen durch den $r$ Hydrothorax P.unktion: $500 \mathrm{ccm}$ bierbraune Flüssigkeit, spezifisches Gewicht 1012, Rivalta chmierkur wird gut vertragen.

3. XI. Aszites geht bedeutend zurück, Hydrothorax r. reicht noch bis zum Angulus scapulae. Leber: scharfrandig, 3 Querfinger unter dem Rippenbogen füh.bar, Milz nicht palpabel.

8. XI. Ikterus bedeutend zurückgegangen. Hydrothorax klinisch und röntgenologisch nicht mehr feststelibar. Aszites noch nachweisbar, aber im Rückgang.

Urinausscheidung normal, Urobilin, Bilirubin in Spuren, Urobilinogen - Leuzin. Tyrosin -

15. X1. Ikterus sehr gering, Aszites kaum noch vorhanden. Leber unverändert, scharfrandig.

Patient ist noch bis zum 14. I. 1922 in Behandlung und Beobachtung. Er hat im ganzen $144 \mathrm{~g}$ Ung. hydrarg. cin. geschmiert. Be der Entlassung Leber unverändert groß, Aszites und Hydrothorax nicht mehr nachweisbar, lkterus nicht mehr vorhanden. Patient ist bis jetzt wiederholt zur Nachuntersuchung erschienen und hat noch eine zweite Schmierkur gemacht. Außer der stationär gebliebenen Lebervergrößerung ist nichts mehr nachweisbar

Urin frei von Gallenfarbstoffen, kein Leuzin und Tyrosin.

Wir haben es also mit zwei ziemlich ähnlichen Fällen zu tun, die beide eine Frühsyphilis hatten und etwa 3 Monate nach Abschluß einer Salvarsankur mit lkterus, Aszites, Hydrothorax und in dem einem $F$ all mit Lebervergrößerung, dem anderen Verkleinerung, erkrankten. Bei dem Hydrothorax ist besonders bemerkenswert, $\mathrm{daB}$ er in dem ersten Fall nur linkseitig, in dem zweiten in der Hauptsache rechtseitig und nur in geringer Menge linkseitig aufgetreten ist. Leuzin und Tyrosin konnte in beiden Fällen nicht nachgewiesen werden, während im 2. Fall zunächst Gallenfarbstoffe vorhanden waren, von diesen im ersten schnell zum Exitus gekommenen nur Bilirubin nachweisbar war.

Auf das histologische Leberbild des ersten Falles, das vollständig übereinstimmt mit jenen Bildern subakuter Leberatrophie, wie sie von Hart, Hanser, Versé u. a. geschildert werden, wollen wir hier nicht näher eingehen, ebenso auch nicht auf die. Erklärung und Deutung des Aszites. Dieses Symptom ist von $\mathrm{Huber}$ und $\mathrm{Ka} u \mathrm{sch}$ $S t r a u B$, Umber, Lyon u. a. genügend beschrieben worden. Uns liegt vor allem daran, auf die sehr interessante Beobachtung hinzuweisen, daß neben dem Aszites in dem einen $F$ all ein streng einseitiger Hydrothorax und schweres Anasarka und in dem anderen auf der einen Seite ein aus. gedehnter, aufder andereneingeringer Hydrothorax vorhanden war. Wir möchten nicht glauben, daB es sich hier, zumal in dem zweiten klinisch ausgeheilten Falle, um ein Symptom handelt, das etwa durch Herzschwäche ausgelöst ist, sondern nehmen vielmehr an, daß der $\mathrm{Hydroth}$ orax und ebenso auch das Anas a rka ihre Ursache in der Lebererkrankung haben, die bei ihrer langen Dauer zu den schweren allgemeinen Stauungserscheinungen geführt hat. Vielleicht spielen bei dem $r$. Hydrothorax direkt von der Leber aus fortgelcitete Ursachen eine Rolle. Es wäre auch möglich, daß toxi iche Gefäßschädigungen, wie sie Vers é beim Aszites vermutet hat, mitgewirkt haben.

Neben dem zweiten ausgeheilten Fall haben wir gerade in der letzten Zeit mehrere Beobachtungen gemacht, wo Patienten im V e rlaufe eines mehr oder weniger schweren Ikterus ein bis mehrere Tage anhaltende Ausscheidung von Leuzin und Tyrosin, das chemisch im Chemischen Laboratorium des Rudolf Virchow-Krankenhauses nachgewiesen wurde, zeigten, ohne dả sich ausgedehntere Störungen bemerkbar machten, während $z$ B in einem Falle eine Frau im Verlaute eines Ikterus, der mit einer zunächst deutlich nachweisbaren Vergrößerung und späteren Verkleinerung der Leber einherging, auf der Höhe des Krankheits- 
prozesses, der unter spezitischer Behandlung abheilte, mehrere Tage eine sehr starke Ausscheidung von Leuzin und Tyrosin aufwies. Die betreffende Patientin ist zur Zeit zu einer Wiederholungskur bei uns und zeigt eine deutliche Verkleinerung der Leber mit einem harten scharfen Rande, während die übrigen Patienten, die vorübergehend einige Tage eine Leuzin- und Tyrosinausscheidung hatten, keinerle Symptome mehr zeigen. Schon früher sind von Bus chke, Buschke und Zernik aus unserer Klinik ähnliche Fälle beschrieben worden. Ausdrücklich sei erwähnt, das wir auch Fälle von verhältnismäßig leichtem lkterus sahen, bei denen eine dauernde Vergrößerung und Verhärtung der Leber zurückblieb, sodaß auch bei leichten Fällen nicht sicher gezeigt ist, daß völlige Heilung eintritt.

Es wäre somit die Frage zu beantworten, ob und welche $\mathrm{Be}$ deutung die Leuzin- und Tyrosinausscheidung, von der wir natürlich wie $G$ öbel den chemisch einwandfreien Nachweis verangen müssen, im Verlaufe eines Ikterus hat. Jedenfalls scheinen doch neben den mit der ganzen Schwere ihrer Symptome auftretenden Fällen des öfteren jetzt solche vorzukommen, die abortiv mit leichten Beschwerden und klinischen Erscheinungen verlaufen und die unser Augenmerk besonders verdienen, da sie quoad restitutionem sicherlich therapeutischer Beeinflussung gut zugänglich sind. Damit ergibt sich aber eine weitere, schon vor langer Zeit von Buschke und seinen Schülern, Zernik und Michael angeschnittene Frage, ob die Leberatrophie überhaupt als ein selbständiges Krankheitsbild behandelt werden darf, oder ob wir nicht vielmehr von leichteren lkterus fließende Uebergänge haben bis zur zirrhot ischen Ausheilung. Unter diesen Umständen wären, was wir bisher als eigene Krankheitsgruppen betrachtet haben, nicht als solche anzusehen, sondern sie würden nur mehr oder weniger leichte bzw. schwere Symptome einer Lebererkrankung darstellen. Diesen Standpunkt $\mathrm{Buschkes}$ teilen auch $\mathrm{Minkowski}$ und neuerdings $\mathrm{Kirch}$ und Freundlich u. a. Die Hauptschwierigkeit, der.wir bei der wichtigen Beurteilung der Schwere des Krankheitsbildes begegnen, ist der exakte Nachweis der Leberfunktionsstörung. Wenn wir auch zahlreiche Fälle auf der einen Seite haben, bei denen im Verlaufe der Erkrankung, wie bei unseren zuletzt erwähnten Beobachtungen, Leuzin und Tyrosin einwandfreier nachgewiesen werden konnten (B us chke, Buschke und Zernik, Strümpell), liegen doch anderseits analog den beiden zuerst beschriebenen Fällen zahlreiche Beobachtungen vor wie z. B. von Lepehne, daB im Verlauf auch einer schweren Lebererkrankung, Leuzin und Tyrosin fehlen können. Und wir schließen uns völlig der Ansicht Umbers an, daß bei abortiven Fällen das Fehlen von Leuzin und Tyrosinkein Beweis gegen eine parenchymatöse Erkrankung der Leber ist.

Es wird vielfach der Erkrankungsprozeß der Leber mit den parenchymatösen Nierenschädigungen verglichen (Buschke und Ze rnik, $\mathrm{Minkowski,} \mathrm{Strümpell),} \mathrm{nur} \mathrm{daß} \mathrm{bei} \mathrm{letzteren} \mathrm{der} \mathrm{Nachweis}$ der Funktionsstörung viel leichter und einfacher ist. Sicherlich tritt das sichtbare Symptom der parenchymatösen Lebererkrankung der Ikterus, viel später in Erscheinung als die ihn bedingenden Veränderungen der Leberzellen. Wir m üs sen wohl an nehmen, da $B$ bei den leichten, ohne nachweisbare Lebervergröße. rung bzw. Verkleinerung einhergehenden lkterus formen die Schädigungen der Leberzellen gering fügig und zumeist reparabel sind und daß es von diesem Stadium aus alle möglichen Uebergänge über die akute bzw. subakute Leberatrophie bis zu den zirchotischen Schrumpfungsprozessen gibt. Das Tempodes Zerfalls wird durch die Schwerederschädigenden Noxe a u d ereinen Seite und die vorhandene Widerstandsfähigkeit bzw. Empfänglichkeit des Orans auf der andern Seite angegeben. Und es dürfte auch hiervon abhängen, ob Leuzin und Tyrosin, die Symptome eines gestörten Leberstoffwechsels, erscheinen oder nicht. Wir können woh! annehmen, daß die Hepatitis bis zu einem gewissen Grenzwert gesteigert werden kann, ohne daß neben dem lkterus schwere Symptome in Erscheinung treten; wird dieser aber überschritten, so bekommen wir das Bild der Leberatrophie, die auch zum Teil noch wenigstens klinisch reparabel sein kann, während anatomisch niemals mehr eine normale Leberzellstruktur in den Regenerationsherden nachweisbar ist. Diese an und für sich schon in ihrer Funktion und in ihrer Widerstandsfähigkeit geschädigten Lebern vertragen selbstverständlich neu auftretende Rezidive schlechter als die nur leicht und abortiv erkrankten Organe.

Somit erscheint uns heute das Bild der Lebererkrankungen vom Ikterus bis zur Zirrhose als ein einheitliches und nur graduelı gegeneinander abgestuftes, und wir müssen in diese Reihe auch alle jenen nur abortiv verlaufenden Fälle mit einreihen, die eins der wenigen nachweisbaren Symptome einer gestörten Leberfunktion aufweisen. Für einen Teil der Fälle ist der Nachweis von Leuzin und Tyrosin verwendbar, ihr Vorkommen hängt aber anscheinend von vielerlei Momenten ab, unter anderem wohl von der Ausdehnung und Schnelligkeit des Zerfalls, sodaß es keineswegs als ein sicheres Diagnostikum verwendbar ist. Wie weit die in letzter Zeit mitgeteilten Prüfungen der Leberfunktion mittels Farbstoffausscheidung (Lepeh ne, R o s e nthal und von Falkenhausen. Hesse und Wörner) uns den Weg erleichtern können, müssen erst weitere Versuche zeigen. Mit dem Grade des ja auch nur symptomatisch auftretenden Ikterus, der aber erst in Erscheinung tritt, wenn die krankhaften Veränderungen der Leber längst eingesetzt haben, können wir zur Feststellung der
Schwere der Erkrankung auch nichts antangen. Auch die übrigen Funktionsprüfungen, z. B. die Lävulose-Probe, lassen uns mehr ode weniger im Stich. So sind wir bei den hepatitischen. Erkrankungen im großen und ganzen daraut angewiesen, durch Vergleich mit trüher gemachten Beobachtungen und auf Grund unserer Erfahrung unsere klinische Diagnose zu stellen.

Stehen wir schon bei der Beurteilung des Krankheitsbildes als solchen ziemlich vielen unbekannten Größen gegenüber, so sind wir aber ganz auf Hypothesen angewiesen, wenn wir die Aetiologie des Prozesses einer Betrachtung unterziehen. So lange wir keine einheitliche Erklärung der Aetiologie der Lebererkrankungen haben, wird sich für jede der Theorien ein Für und Wider anführen lassen. Sicherlich gibt es eine Reihe von Fällen, von denen man mit Umber eine cholangene Ursache annehmen kann, des weiteren dürfte der Beweis dafür erbracht sein, daß die schlechte Ernährung in und kurz nach dem Kriege zu Störungen im Lebergleichgewicht geführt hat ( $M$ i n kowski), wofür $u$. a. von Jacobsohn und $S$ klarz aus unserer Klinik experimentell der Nachweis erbracht worden ist. Des ferneren spielt sicherlich noch eine Reihe anderer exo- und endogener $\mathrm{Zu}$ fallsmomente für einen Teil der Fälle eine entscheidende Rolle.

Wenn wir uns hier aber insbesondere den Fällen von Lebererkrankungen bei Syphilis mit und ohne spezifische Behandlung ozuwenden, so stehen wir der Frage gegenüber: was ist bei der Erkrankung der Leber auf das Konto der Syphilis und was a uf das der Behandlung zu setzen?

Buschke, Michael, Buschke und Zernik haben an einer Reihe von Beispielen auseinandergesetzt, daß das, was für den gewöhnlichen lkterus, auch für den lcterus syphiliticus praecox gilt, sodaß er als eine parenchymatös $\mathrm{e}$ Lebererkrankung und damit der Leberatrophie gleichartige und von ih graduell unterschiedene Erkrankung anzusehen ist und nehmen für beide eine toxisch-spezifische Ge nese an. In den vielen daraut hin gerichteten Untersuchungen sind bei diesen Lebererkrankungen, soweit sie untersucht werden konnten, Spirochäten in der Leber nicht nachgewiesen worden $(F$ i s ch e r, Buschke und Fischer, Veszpremy und Kanitz). Hieraus dürfte wohl der Schluß berechtigt erscheinen, daß der. Iebenden Spirochäte als solcher im wesentlichen kein Wert fü die Aetiologie beizumessen ist, daß es vielmehr dievon de Spirochäte gebildeten oder bei ihrem Zerfall gelie ferten Toxine sind, die sich mehr als in anderen Organen in der Leber anhäufen und hier zu der Erkrankung führen.

Wie manche Fälle, u. a. die von $B$ us ch ke beobachteten, zeigen, kann im AnschluB an eine energische $\mathrm{Hg}-\mathrm{Kur}$ atch eine Lèberschädigung auftreten, als deren Ursache wir dann den gehäuften Untergang von Spirochäten und das Erscheinen der toxisch spezifischen Noxe annehmen müssen. Auf der anderen Seite ist cs eine unleugbare Tatsache, daß, seit wir in ausgedehnter und oft wenig sachgemäßer Weise die $\mathrm{Salvarsanbehandlung}$ haben, die leichten und schweren Lebererkrankungen zugenommen haben (Arndt). Dieses Zusammentreffen kann man doch keineswegs als Zufall bezeichnen, sondern muß anerkennen, daß dem Salvarsan in gewissen Fällen allein oder im Verein mit anderen Komponenten die Schuld zuzuschieben ist.

Auf der einen Seite ist es natürlich leicht erklärlich daß eine an sich bisher durch kein Moment geschädigte Leber die Verabtolgung des Salvarsans nicht verträgt und es auf diesem Wege zu einer Schädigung kommt, die wir als reine Salvarsanschädigung bezeichnen müssen. Wieviel von allen Fällen auf dieses Konto zu setzen ist, kann man allerdings noch nicht bei der Unzulänglichkeit unserer Methoden nachweisen. Für einen anderen $\mathrm{T}$ e il stehen auch wir aut dem Standpunkte, daß es nicht alle in das $S$ alvarsan ist, das die Erkrankung hervorruft, sondern daß es nur de $r$ a us lö send e $F$ a k tor ist, in dem z. B. durch gehäuften Spirochätenzertall reichlich Toxinc frei werden, die zu der Lebererkrankung führen oder zum. Teil (vgl. unten) eine Provokation einer spezifischen Erkrankung des vegetativen Nervensystems in diesem Gebiet. Des weiteren darf man woh annehmen, $\mathrm{daB}$ es auch in der Leber durch die provokatorisch medikamentöse Beeinflussung seltener beim $\mathrm{Hg}$, jedoch häufiger durch das Salvarsan zu einer parenchymatösen Störung kommt, die mit den Erscheinungen der Herxheimerschen Reaktion vielleicht auf eine Stufe zı stellen wären und die uns zum großen Teil die Fälle liefert, die dic abortiven Erkrankungserscheinungen bieten, und die ebenso wie die reiı syphilitisch-toxischen für die Therapie erfolgreich und aussichtsreich sind.

Nicht unerwähnt möchten wir auf Grund einer kürzlichen an anderer Stelle ausführlich $\mathrm{zu}$ publizierenden Beobachtung lassen, daß vielleicht in manchen Fälle n daran zu denken ist, daß die Symptome der Lebererkrankung und vielleicht auch ebenso die der eigentlich syphilitischen, im Frühstadium mit starker Absonderung von Eiweiß und geringer von Harnzylindern einhergehenden Nierenstörung, wie wir es für manche Salvarsanexantheme annehmen müssen, durch nervösen EinfluB bedingt sind, durch eine spezifische Erkrankung im sympathischen System, daher vielleicht die oft zauberhaft schnelle therapeutische Beeinflussung durch spezifische Medikamente.

Auf Grund der auseinandergesetzten Anschauung über die Aetilogie richten wir auch unsere Therapie ein, indem wir nach kurzem Abwarten je nach dem Eindruck, den wir von dem einzelnen Fall haben, mehr oder weniger energisch mit Quecksilber behandeln und, 
wenn wir mit diesem nicht zum Ziele kommen, in manchen Fällen auch mit kleinen Salvarsandosen symptomatisch vorgehen.

Zusanmenfassung: 1. Neben dem Aszites ist als begleitende Momente der Leberatrophie auf Anasarka und Hydrothorax zu achten.

2. Es gibt eine Reihe von Fällen von schwerer Leberatrophie, die in klinische Heilung übergehen können; daneben finden sich öfters abortiv auftretende Erkrankungsformen.

3. Leuzin und Tyrosin zeigen im Verlauf eines Ikterus eine schwerere Leberstörung an, sind aber kein regelmäßig vorkommendes Symptom selbst bei schwereren Affektionen.

4. Erkrankungsformen vom Ikterus bis zur Leberatrophie mit evtl. partieller Zirrhose sind als eine einheitliche, nur graduell verschiedene Erkrankung anzusehen, die wahrscheinlich wesentlich auf eine parenchymatöse Schädigung der Leberzellen zurückzuführen ist.

5. Die parenchymatöse Schädigung der Leber im Verlaufe einer Syphilis mit und ohne Behandlung ist meistens als durch eine toxischspezifische Noxe bedingt za betrachten. Der Behandlung, speziell dem Salvarsan, kommt nur in einem Teil der Fälle eine primär-ätiologische Ursache zu, öfter dagegen ist es als sekundär auslösendes Moment anzusehen. Vielleicht spielt das vegetative Nervensystem bei der Entstehung dieser Affektion auch eine gewisse Rolle.

6. Selbst schwere Formen von Leberatrophie auf syphilitischer Basis können der Therapie zugänglich sein.

A rndt, M. K1. 1922 Nr. 8/9. - Bange, Diss. 1914 Berlin. - Buschke, B. kl. W. 1910. - Buschke und Fis cher, Arch. f. Derm. u. Syph. 82, H. I. - Buschke und

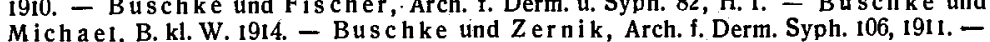

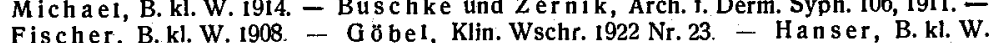
Fischer, B. kl. W. 1908. - Go obel, Klin. Wschr. 1922 Nr. 23. - H anser, B. Kl. W. 1921. - Hart, M. Kl. 1921. - Hesse und Worner, Klin. Wschr. 1922 Nr. 23. - H I a va, Prag. m. Wschr 1882. - Huber und Ka us ch, B. kt. W. 1920. - Ja co obs o hn und Sklarz, M. KL. 1921 u. 1922. - Kirch und Freundilich, Arch. f. Derm. u. Syph. 136, 1921.Lepehne, B. kL. W. 1921; D. m. W. 1921. - Lyon, M. KI. 1921. - March and, Beitr. z. path. Anat. hem. hausen, B.k.W. 192. - Sey rarth, D W.W. 1920. - Strütmpell, 1878. - U Umber, B. kl. W. 1920; D. m. W. 1920. - Versé, B. kl. W. 1920. - Veszpremy und $\mathrm{K}$ a n i $z$, Arch. f. Derm. u. Syph. 88, 1907 . Wai de y er, Virch. Arch $43,1868$. 